\title{
PROPOSITIONAL CALCULUS AND REALIZABILITY
}

\author{
BY
}

GENE F. ROSE

In [13], Kleene formulated a truth-notion called "realizability" for formulas of intuitionistic number theory $\left({ }^{1}\right)$. David Nelson $\left({ }^{2}\right)$ showed that every number-theoretic formula deducible in the intuitionistic predicate calculus $\left(^{3}\right.$ ) (stated by means of schemata, without proposition or predicate variables) from realizable number-theoretic formulas is realizable. In particular, then, every formula in the symbolism of the predicate calculus (with proposition and predicate variables) which is provable in the intuitionistic predicate calculus has the property that every number-theoretic formula (in the number-theoretic symbolism without proposition or predicate variables) which comes from it by substitution is realizable. This property was applied by Kleene to demonstrate that certain formulas provable in the classical predicate calculus are unprovable in the intuitionistic predicate calculus $\left({ }^{4}\right)$.

The question naturally arises whether the converse of Nelson's result holds. That is to say, is an arbitrary formula of the predicate calculus provable whenever every number-theoretic formula obtained from it by substitution is realizable? If this question could be answered in the affirmative, we should have a completeness theorem for the intuitionistic predicate calculus $\left(^{(5)}\right.$.

Received by the editors August 14, 1952.

(1) A familiarity with the fundamental results pertaining to this concept is presupposed. For this purpose, the reader is referred to the above paper or to $[17, \S 82]$.

The conjecture which is disposed of in this paper was proposed by Kleene in correspondence in November 1941, and was the only one of an early group of conjectures about realizability which was not settled by 1945 . It was discussed by him in a paper before the Princeton Bicentennial Conference on the Problems of Mathematics in December 1946 (unpublished). The author took up the investigation in 1947, following a suggestion by Kleene that Jaskowski's matric treatment of the Heyting propositional calculus [10] might provide a basis for attacking that part of the problem which concerns the propositional calculus. The solution by a counterexample, presented here, was obtained in February 1951.

The material in this paper is included in Jaskowski's truth-tables and realizability, a thesis submitted in partial fulfilment of the requirements for the degree of Doctor of Philosophy at the University of Wisconsin and accepted in February 1952. In the thesis, the proofs of the following lemmas and theorems are given in greater detail: $3.2,4.5,4.6,4.7,4.8,5.1,5.2,5.3,5.4$, 6.1, 7.1, 7.2.

(2) Cf. Nelson [23, Theorem 1] or Kleene [17, §82, Theorem 62(a)].

(3) Cf. $[8 ; 9 ; 2]$.

(4) Cf. $[13, \S 10]$. Other demonstrations were given later: cf. [14;17, §80;22].

(5) For the classical predicate calculus, there is the well known completeness theorem of Gödel [4]. For the intuitionistic predicate calculus, on the other hand, no completeness theorem was known until 1949 , when one not closely connected with the logical interpretation was found by Henkin [7] as a kind of converse of a result of Mostowski [22]. 
The position of the intuitionists that mathematical reasoning can never be completely formalized (cf. [8]) found subsequent confirmation in Gödel's celebrated incompleteness theorem for number theory [5], which applies to both classical and intuitionistic systems. However, this does not preclude the possibility that, as in the corresponding case for classical reasoning, an (effectively) formalizable set of principles might embrace all forms of intuitionistic reasoning which entail only the subject-predicate structure (or perhaps only the propositional form) of the propositions involved. If the stated conjecture should be true, the Heyting calculus would already give such a characterization; if false, a counterexample might point the way either to finding a more explicit intuitionistic interpretation or to extending the calculus to include additional principles acceptable in the intuitionistic predicate logic. Consequently, the conjecture was investigated with no clear indication a priori as to its truth or falsity.

We now know that it is false; and a counterexample can be presented briefly (cf. Theorem 6.1 below). This counterexample, however, was discovered only after prolonged, systematic attempts to establish the conjecture for various classes of formulas, beginning with the propositional calculus, and to abstract some general principles. This paper contains, besides the decisive example, the principal results of the investigation which culminated in it. Inasmuch as the counterexample appeared within the propositional calculus, the part of the problem relating to the full predicate calculus was never reached. The fact that the completeness conjecture holds for the part of the intuitionistic propositional calculus without implication (cf. Theorem 7.5 below) emphasizes the special difficulty connected with the intuitionistic interpretation of implication.

The counterexample is established only classically $\left({ }^{6}\right)$. Hence, rather than immediately seeking an extension of the intuitionistic propositional calculus, we should examine the interpretation along the following lines.

Kleene reports $\left({ }^{7}\right)$ that his current work on realizability of formulas with function variables, as proposed in [16, end \$2], may cast new light on the intuitionistic interpretation of number-theoretic formulas containing implication or negation.

Moreover, one might seek to establish completeness by allowing the substitution of formulas from the enlarged class containing function variables. A complete predicate calculus should provide for just those methods of predicate reasoning which are valid in any part of intuitionistic mathematics. One can start with a given notion of formula with an interpretation, and consider the class of all predicate formulas valid for reasoning with these formulas. Then, as the class of formulas is increased, the class of predicate

${ }^{(6)}$ All results in this paper are established intuitionistically, except those which are indicated as only having been established classically.

(7) In December 1951. 
formulas does not increase. The "complete predicate calculus" can be defined (noneffectively) as the inner limiting set of the sets of valid formulas upon repeating this process for all conceivable extensions of the "formal context." It was a major discovery of Gödel [4] that, in the case of classical logic, one already obtains the inner limiting set after including the number-theoretic formulas, as one might expect from the earlier results of Löwenheim [18] and Skolem [25]. In analogy to the classical case, it was natural that the first attempt at a completeness property for the intuitionistic predicate calculus should be by a number-theoretic interpretation; but it must be kept in mind that this is not the only possibility.

\section{PART I}

1. Propositional calculus. We shall refer to the variables and formulas of the number-theoretic formal system of $[13, \S 4]$ as $n$-variables and $n$-formulas. We now consider the propositional calculus as a separate formal system, and refer to its variables and formulas as p-variables and p-formulas. Let us define a p-formula to be realizable if and only if every $n$-formula obtained from it by substituting $\mathrm{n}$-formulas for its $\mathrm{p}$-variables is realizable. The set of

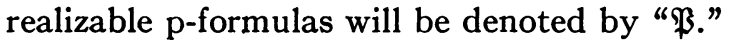

A propositional calculus will be called consistent with respect to realizability if its provable $\mathrm{p}$-formulas form a subset of $\mathfrak{B}$; complete with respect to realizabil$i t y$ if the set of its provable p-formulas contains $\mathfrak{B}$. In investigating the conjecture that the Heyting propositional calculus is complete with respect to realizability, we shall treat this calculus as it is set forth by modus ponens, the substitution rule, and the following axioms (where $\mathrm{a}, \mathrm{b}$ and $\mathrm{c}$ are distinct p-variables):

(1a) $a \supset(b \supset a)$.

(3) $\quad a \supset(b \supset a \& b)$.

(5a) $\mathrm{a} \supset \mathrm{a} \vee \mathrm{b}$.

(5b) bつa $\mathrm{ab}$.

$$
(\mathrm{a} \supset \mathrm{b}) \supset((\mathrm{a} \supset\urcorner \mathrm{b}) \supset\urcorner \mathrm{a}) \text {. }
$$

The set of p-formulas provable in the Heyting propositional calculus will be denoted by "S."

The postulates of the classical propositional calculus differ from those of Heyting's in that the last axiom above is replaced by $77 \mathrm{a} \supset \mathrm{a}$. The set of p-formulas provable in this calculus will be called " $\mathfrak{U}$."

2. Matrices. Our use of matrices (or truth-tables) for the representation of sets of p-formulas is based directly on [20], [10], and [21, p. 3], and ultimately on [19] and [24]. By a (finite) matrix $M$, let us understand a system consisting of a finite set of elements closed under each of three binary 
operations $\supset_{M}, \&_{M}, \bigvee_{M}$ and one unary operation $\rceil_{M}$, and having a nonempty subset of designated elements. An evaluating function with respect to a matrix $M$ is any homomorphic mapping of the p-formulas into $M$ such that $\supset, \&, V$, and $\rceil$ (regarded as formal operations) correspond respectively to $\supset_{M}, \&_{M}, \vee_{M}$, and $\rceil_{M}$. A p-formula fulfils $M$ if and only if it is mapped into a designated element by every evaluating function with respect to $M$. A matrix $M$ is regular if and only if, for arbitrary matrix elements $e$ and $f, f$ is designated whenever $e$ and $e \supset_{M} f$ are designated. A matrix $M$ is a characteristic matrix for a set $\mathfrak{F}$ of $\mathrm{p}$-formulas if and only if $\mathfrak{F}$ is the set of $\mathrm{p}$-formulas which fulfil $M$. A sequence $\left\{M_{i}\right\}(i=0,1, \cdots)$ of matrices is regular if and only if each $M_{i}$ is regular and there is an effective process by which, for all $i, M_{i+1}$ is defined in terms of $M_{i}$. Such a sequence is a characteristic sequence for a set $\mathfrak{F}$ of $\mathrm{p}$-formulas if and only if $\mathfrak{F}$ is the set of $\mathrm{p}$-formulas which fulfil every matrix in the sequence.

Gödel [6] has shown that $\mathfrak{S}$ has no regular characteristic matrix. Jaśkowski [10], however, has exhibited a characteristic sequence for $\mathfrak{S}$. In Theorem 7.6, we shall show that $\mathfrak{P}$ has no regular characteristic matrix; the existence of a characteristic sequence for $\mathfrak{B}$ remains an open question.

We shall devote the remaining sections of Part I $(\$ \$ 3-5)$ to an inessentially modified $\left({ }^{8}\right)$ presentation of Jaśkowski's above-mentioned result, for which a detailed proof is nowhere available in the literature. The results on realizability follow in Part II.

\section{Simple conjunctions and simple implications.}

3.1. Definitions. We shall denote finite, possibly empty, sequences of $\mathrm{p}$-formulas (of $\mathrm{n}$-formuas) by capital Greek letters. For $\mathrm{p}$-formulas (for $\mathrm{n}$-formulas) $\Delta, \mathrm{A}$, " $\Delta \vdash \mathrm{A}$ " will mean that $\mathrm{A}$ is deducible from $\Delta$ in the Heyting propositional calculus (in the intuitionistic predicate calculus with equality and the Peano axioms). By "A $\dashv \vdash \mathrm{B}$ " we shall mean that $A \vdash B$ and $B \vdash A$. We shall use $A \sim B$ as an abbreviation for $(A \supset B) \&(B \supset A)$, saying that $\mathrm{A}$ is equivalent to $\mathrm{B}$ if $\vdash \mathrm{A} \sim \mathrm{B}$.

We write " $\prod_{i \leqq n} A_{i}$ " for $A_{0} \& \cdots \& A_{n}$, and " $\sum_{i \leqq n} A_{i}$ " for $A_{0} \vee \cdots$ $\vee \mathrm{A}_{n}$, where $\mathrm{A}_{0}, \cdots, \mathrm{A}_{n}$ are $\mathrm{p}$ - or $\mathrm{n}$-formulas called the members of the conjunction or disjunction respectively $\left({ }^{9}\right)$. For any $j \leqq n$, the result of replacing $\mathrm{A}_{j}$ by $\mathrm{B}$ in $\prod_{i \leqq n} \mathrm{~A}_{i}$ will be denoted by " $j(\mathrm{~B}) \prod_{i \leqq n} \mathrm{~A}_{i}$."

Henceforth, lower-case latin letters will be used, in connection with p-formulas, only for the designation of p-variables which, unless otherwise specified, are not necessarily distinct.

A simple conjunction is a p-formula $\mathrm{K}$ of the form $\prod_{i \leqq n} \mathrm{~A}_{i}$ where each $\mathrm{A}_{i}$ has one of the following forms $\left({ }^{10}\right)$ : (i) a, (ii) $\neg \mathrm{a}$, (iii) $\mathrm{a} \supset \mathrm{b}$, (iv) $\mathrm{a} \supset \mathrm{b} \vee \mathrm{c}$,

(8) The modifications will be noted as they occur.

( $)$ More specifically, $\prod_{i \leqq n} A_{i}$ is $A_{0}$ for $n=0$, and $\left(\cdots\left(\left(A_{0} \& A_{1}\right) \& A_{2}\right) \cdots\right) \& A_{n}$ for $n \geqq 2$. Similarly for $\sum_{i \leqq n} A_{i}$.

(10) $\mathrm{a} \supset \mathrm{b} \bigvee \mathrm{c}$ means $\mathrm{a} \supset(\mathrm{b} \vee \mathrm{c}$ ), and a \& b c means (a \& b) つc. 
(v) a \& bつc, (vi) (aつb)つc. We define the degree $d(\mathrm{~K})$ of $\mathrm{K}$ to be the number of members which have form (vi).

A simple implication is a $\mathrm{p}$-formula of the form $\mathrm{K} \supset \mathrm{z}$ where $\mathrm{K}$ is a simple conjunction and $z$ is any p-variable $\left({ }^{11}\right)$.

If $\mathrm{K}$ is the simple conjunction $\prod_{i \leqq n} \mathrm{~A}_{i}, \mathrm{P}$ is the simple implication $\mathrm{K} \supset \mathrm{z}$ and $\mathrm{A}_{j}$ is $\left(\mathrm{a}_{j} \supset \mathrm{b}_{j}\right) \supset \mathrm{c}_{j}$, then we denote $j\left(\mathrm{a}_{j} \&\left(\mathrm{~b}_{j} \supset \mathrm{c}_{j}\right)\right) \prod_{i \leqq n} \mathrm{~A}_{i}$ by "K $\mathrm{K}^{j}$ " and $\mathrm{K}^{j} \supset \mathrm{b}_{j}$ by " $\mathrm{P}^{j}$."

3.2. Lемма. If $\mathrm{P}$ is a p-formula of the form $\prod_{i \leqq n} \mathrm{~B}_{i} \supset z$ where each $\mathrm{B}_{i}$ is an implication or a negation, then $\mathrm{P}$ is interdeducible with a simple implication.

Proof. Let $\mathrm{P}$ fulfil the hypothesis of the lemma. For each $\mathrm{B}_{i}$, define $p\left(\mathrm{~B}_{i}\right)$ to be 0 or the number of (occurrences of) p-variables and 7 's in $B_{i}$ according as $\mathrm{B}_{i}$ has one of the forms (ii)-(vi) or not. Let $q(\mathrm{P})$ be $\max _{i \leqq n} p\left(\mathrm{~B}_{i}\right) ; m(\mathrm{P})$, the number of $\mathrm{B}_{i}$ 's such that $p\left(\mathrm{~B}_{i}\right)=q(\mathrm{P})$. The proof is completed by showing that $1^{\circ}$ the lemma holds when $q(\mathrm{P})=0$ and $2^{\circ}$ if $q(\mathrm{P})>0$, then there exists a p-formula $\mathrm{Q}$, interdeducible with $\mathrm{P}$, such that either $q(\mathrm{Q})<q(\mathrm{P})$ or else $q(\mathrm{Q})=q(\mathrm{P})$ and $m(\mathrm{Q})<m(\mathrm{P})$.

3.3 Theorem. Every p-formula is interdeducible with a simple implica$\operatorname{tion}\left({ }^{12}\right)$.

Proof. Let $\mathrm{P}$ be an arbitrary $\mathrm{p}$-formula, $\mathrm{x}$ a $\mathrm{p}$-variable not in $\mathrm{P}$. Then $\mathrm{P} \dashv \vdash(\mathrm{P} \supset \mathrm{x}) \supset \mathrm{x} \dashv \vdash[3.2]$ a simple implication.

4. The matrix-sequence $\left\{J_{i}\right\}$. Let us define the set $\mathfrak{X}$ thus. $1^{\circ}$ If $x$ is a natural number, then $x \in \mathfrak{X}$. $2^{\circ}$ For any $d \geqq 1$, if $x_{1}, \cdots, x_{d} \in \mathfrak{X}$, then $\left(x_{1}, \cdots, x_{d}\right) \in \mathfrak{X}$. For any $d \geqq 1$ and any $x \in \mathfrak{X}$, " $x^{(d)}$ " denotes the $d$-tuple $(x, \cdots, x) .3^{\circ}$ All elements of $\mathfrak{X}$ are given by $1^{\circ}$ and $2^{\circ}$.

The partial ordering relation $\prec$ in $\mathfrak{X}$ is defined thus. $1^{\circ}$ For any $d \geqq 1$ and any $i(1 \leqq i \leqq d), x_{i} \prec\left(x_{1}, \cdots, x_{d}\right) .2^{\circ}$ For any $d \geqq 1$ and any $i(1 \leqq i \leqq d)$, if $x<x_{i}$, then $x<\left(x_{1}, \cdots, x_{d}\right) .3^{\circ}$ For any $x, y$ in $\mathfrak{X}, x<y$ only as required by $1^{\circ}$ and $2^{\circ}$.

Note that, for any $x$ in $\mathfrak{X}, x$ is distinct from $x^{(1)}$, which is $(x)$.

4.1. We define an infinite set $\{M\}$ of matrices in such a way that, for each member, the elements are in $\mathfrak{X}$ and the designated element is unique. For any $M$ in $\{M\}$, we denote the designated element by " $b_{M}$ " and the (possibly empty) set of undesignated elements by " $A_{M}$. " Thus:

4.1.1. The matrix $L_{0}$ whose sole element is 0 is in $\{M\}\left({ }^{13}\right)$.

(11) The class of simple implications differs from Jaskowski's class of regular formulas in that a member of the premise of a regular formula may have one of the additional forms $\mathrm{a} \vee \mathrm{b} \supset \mathrm{c}, \neg \mathrm{a} \supset \mathrm{b}, \mathrm{a} \supset(\mathrm{b} \supset \mathrm{c}), \mathrm{a} \supset \mathrm{b} \& \mathrm{c}, \mathrm{a} \supset \overline{\mathrm{b}}$, but it may not be a variable.

${ }^{12}$ Jaskowski states without proof that any formula is interdeducible with a formula of the form $R_{0} \& \cdots \& R_{n}$ where each $R_{i}$ is regular.

(13) This matrix corresponds to Jaśkowski's $\mathbb{R}_{1}$. 
4.1.2. If $M \in\{M\}$, then $\Gamma(M) \in\{M\}$, where $\Gamma(M)$, which for convenience we denote by " $N$," is defined thus. Let $b_{N}=\operatorname{def} b_{M}$, and $A_{N}=\operatorname{def} A_{M}$ $+\left\{a_{N}\right\}$ where $a_{N}$ is the least natural number $n$ such that for no element $x$ of $M$ is $n=x$ or $n<x$. Now for any $x$ in $M$, let $\alpha_{M}(x)$ be $x$ or $a_{N}$ according as $x \in A_{M}$ or $x=b_{M}$. We define the operations of $N$ in terms of those of $M$, using the following tabular form.

\begin{tabular}{|c|c|c|}
\hline$x \supset_{N} y$ & $y=b_{N}$ & $y=\alpha_{M}(v)$ \\
\hline$x=b_{N}$ & $x \supset_{M} y$ & $\alpha_{M}\left(x \supset_{M} v\right)$ \\
\hline$x=\alpha_{M}(u)$ & $u \supset_{M} y$ & $u \supset_{M} v$ \\
\hline$x \&_{N} y$ & $y=b_{N}$ & $y=\alpha_{M}(v)$ \\
\hline$x=b_{N}$ & $x \&_{M} y$ & $\alpha_{M}\left(x \&_{M} v\right)$ \\
\hline$x=\alpha_{M}(u)$ & $\alpha_{M}\left(u \&_{M} y\right)$ & $\alpha_{M}\left(u \&_{M} v\right)$ \\
\hline$x \vee_{N} y$ & $y=b_{N}$ & $y=\alpha_{M}(v)$ \\
\hline$x=b_{N}$ & $x \bigvee_{M} y$ & $x \bigvee_{M} v$ \\
\hline$x=\alpha_{M}(u)$ & $u \bigvee_{M} y$ & $\alpha_{M}\left(u \bigvee_{M} v\right)$ \\
\hline$\eta_{N} x$ & $\begin{array}{l}{ }_{M}\left(\neg_{M} x\right) \\
\rceil_{M} u \text { if } x\end{array}$ & $x=b_{N}$, \\
\hline
\end{tabular}

The $n$-fold iteration of the operation $\Gamma$ will be denoted by " $\Gamma^{n}$."

4.1.3. If $d \geqq 1$ and, for all $i(1 \leqq i \leqq d), M_{i} \in\{M\}$, then $\left(M_{1}, \cdots, M_{d}\right)$ $\in\{M\}$, where $\left(M_{1}, \cdots, M_{d}\right)$, which for convenience we denote by " $P$," is defined thus. The elements of $P$ are just those ordered $d$-tuples $\left(x_{1}, \cdots, x_{d}\right)$ such that, for all $i(1 \leqq i \leqq d), x_{i} \in M_{i}$. The designated element $b_{P}$ is $\left(b_{M_{1}}, \cdots, b_{M_{d}}\right)$. The operations of $P$ are given by

$$
\begin{gathered}
\left(x_{1}, \cdots, x_{d}\right) \circ_{P}\left(y_{1}, \cdots, y_{d}\right)=\operatorname{def}\left(x_{1} \circ_{M_{1}} y_{1}, \cdots, x_{d} \circ_{M_{d}} y_{d}\right), \\
\neg_{P}\left(x_{1}, \cdots, x_{d}\right)=\operatorname{def}\left(\neg_{M_{1}} x_{1}, \cdots, \neg_{M_{d}} x_{d}\right),
\end{gathered}
$$

where "o" denotes $\supset, \&$, or $\vee\left({ }^{14}\right)$. For any $d \geqq 1$ and any $M$ in $\{M\}$, “ $M^{(d)}$ " denotes the $d$-tuple $(M, \cdots, M)$.

Note that, for any $M$ in $\{M\}, M$ is distinct from $M^{(1)}$.

4.1.4. All matrices in $\{M\}$ are given by 4.1.1.-4.1.3.

${ }^{(14)}$ This operation replaces Jaskowski's iterated binary product. As a result, our $\{M\}$ differs from his corresponding $\left(\Omega_{1}\right)_{\pi r}$. However, there is a biunique mapping of $\{M\}$ onto $\left(\Omega_{1}\right)_{\Pi \Gamma}$ such that corresponding matrices are isomorphic. 
4.2. For all $n>0$, we define $L_{n}$ to be $\Gamma^{n}\left(L_{0}\right)$. The sequence $\left\{L_{n}\right\}$ $(n=0,1, \cdots)$, which is clearly a subset of $\{M\}$, plays an important part in our investigation. The elements of $L_{n}$ are $0, \cdots, n$. The matrix $L_{1}$ is the usual two-valued logic; consequently (cf. [24, pp. 169-171]) it is a regular characteristic matrix for $\mathfrak{A}$. For $n>0$, the operations of $L_{n}$ are given by

$$
\begin{aligned}
& i \supset_{L_{n}} j=\left\{\begin{array}{l}
j \text { if } i=0 \text { or if } i>j>0, \\
0 \text { otherwise; }
\end{array}\right. \\
& i \&_{L_{n}} j=\left\{\begin{array}{l}
j \text { if } i=0 \text { or if } i>j>0, \\
i \text { otherwise; }
\end{array}\right. \\
& i \bigvee_{L_{n}} j= \begin{cases}i \text { if } i=0 \text { or if } i>j>0, \\
j \text { otherwise; }\end{cases} \\
& \rceil_{L_{n}} i= \begin{cases}0 \text { if } i=1, \\
1 \text { otherwise. }\end{cases}
\end{aligned}
$$

4.3. The sequence $\left\{J_{i}\right\}(i=0,1, \ldots)$ also is a subset of $\{M\}$. This sequence corresponds to Jaśkowski's $\left\{\Im_{i}\right\}(i=0,1, \cdots)$. Its members are defined by recursion thus: $J_{0}=\operatorname{def} L_{1}, J_{i+1}=\operatorname{def} \Gamma\left(J_{i}^{(i+1)}\right)$. In $\S 5$, we shall prove that $\left\{J_{i}\right\}$ is a regular characteristic sequence for $\mathfrak{W}$. The following lemmas give some properties of $\{M\}$ which will facilitate this proof. We shall continue to use " $N$ " to denote $\Gamma(M)$ and " $P$ " to denote $\left(M_{1}, \cdots, M_{d}\right)$.

4.4. Lemma. For each matrix $M$ of $\{M\}$,

$$
\begin{aligned}
x \supset_{M} x & =x \supset_{M} b_{M}=x \vee_{M} b_{M}=b_{M} \vee_{M} x=b_{M}, \\
b_{M} \supset_{M} x & =b_{M} \&_{M} x=x \&_{M} b_{M}=x .
\end{aligned}
$$

Corollary. Each matrix in $\{M\}$ is regular.

4.5. Lemma. If $M \in\{M\}$ and $x, y \in M$, then $x \supset_{N} y=x \supset_{M} y$.

4.6. LeмMA. For any matrices $M_{1}, \cdots, M_{d}$ in $\{M\}$, let $v_{1}, \cdots, v_{d}$ be arbitrary evaluating functions with respect to $M_{1}, \cdots, M_{d}$; w be the evaluating function with respect to $P$ such that, for any p-variable $\mathrm{q}, w(\mathrm{q})$ $=\left(v_{1}(\mathrm{q}), \cdots, v_{d}(\mathrm{q})\right)$. Then for any p-formula $\mathrm{P}, w(\mathrm{P})=\left(v_{1}(\mathrm{P}), \cdots, v_{d}(\mathrm{P})\right)$.

4.7. Lemma. For any matrix $M$ in $\{M\}$, let $v$ be an arbitrary evaluating function with respect to $N$; w be the evaluating function with respect to $\Gamma\left(N^{(d)}\right)$ such that $w(\mathrm{q})=v(\mathrm{q})^{(d)}$. Then, for any $p$-formula $\mathrm{P}, w(\mathrm{P})=v(\mathrm{P})^{(d)}$.

4.8. Lemma. For any matrix $M$ in $\{M\}$, let $v$ be an arbitrary evaluating function with respect to $M$; w be the evaluating function with respect to $N$ such that $w(q)=\alpha_{M}(v(q))$. Then, for any p-formula A with one of forms (ii)-(v), $w(\mathrm{~A})=v(\mathrm{~A})$. 


\section{Proof that $\left\{J_{i}\right\}$ is a regular characteristic sequence for $\mathfrak{S}$.}

5.1. Lemma. If $\mathrm{P}$ is an axiom of the Heyting propositional calculus and $M \in\{M\}$, then $\mathrm{P}$ fulfils $M$.

5.2. Theorem. For any matrix $M$ in $\{M\}$ and p-formulas $\Delta, \mathrm{P}$ such that $\Delta \vdash \mathrm{P}:$ if each member of $\Delta$ fulfils $M$, then so does $\mathrm{P}\left({ }^{15}\right)$.

Proof. It can be shown that the conclusion holds under each of the following cases: $1^{\circ} \mathrm{P}$ is an axiom. $2^{\circ} \mathrm{P} \in \Delta .3^{\circ} \Delta \vdash \mathrm{A}, \Delta \vdash \mathrm{A} \supset \mathrm{P}$ and the conclusion holds for $\mathrm{A}$ and for $\mathrm{A} \supset \mathrm{P} .4^{\circ} \Delta \vdash \mathrm{A}, \mathrm{P}$ results from $\mathrm{A}$ by substitution and the conclusion holds for A.

If $M$ is a matrix, $\mathrm{K} \supset \mathrm{z}$ a simple implication (cf. 3.1 ), then a normal (refuting) evaluation of $\mathrm{K} \supset \mathrm{z}$ with respect to $M$ is any evaluating function with respect to $M$ which maps each member of $\mathrm{K}$ into $b_{M}$ and $\mathrm{z}$ into $A_{M}$.

5.3. Lemma. Let $P$ be a simple implication $\mathrm{K} \supset$ z. Then either $\mathrm{P}$ has a normal evaluation with respect to $J_{d(\mathrm{~K})}$, or $\vdash \mathrm{P}\left({ }^{16}\right)$.

Proof. Let $\mathrm{K}$ be $\prod_{i \leqq n} \mathrm{~A}_{i}$, and $s(\mathrm{~K})$ be the number of (occurrences of) p-variables and 7 's in $K$. The proof is by exhaustive cases 5.3.1-5.3.3, with the induction hypothesis that the lemma holds for all simple implications $\mathrm{K}^{\prime} \supset \mathrm{z}^{\prime}$ such that either $1^{\circ} d\left(\mathrm{~K}^{\prime}\right)=d(\mathrm{~K})-1$ or $2^{\circ} d\left(\mathrm{~K}^{\prime}\right)=d(\mathrm{~K})$ and $s\left(\mathrm{~K}^{\prime}\right)$ $<s(\mathrm{~K})$. In each case, " $d$ " denotes $d(\mathrm{~K})$.

5.3.1. Suppose that no member of $\mathrm{K}$ is a p-variable. If $d(\mathrm{~K})=0$, a normal evaluation of $\mathrm{P}$ with respect to $J_{0}$ can be readily constructed. If $d(\mathrm{~K})=d>0$, consider two alternatives for the $\mathrm{P}^{i}$ 's.

Suppose that a $\mathrm{P}^{j}$ fulfils $J_{d-1}$. For such a $j$, inasmuch as $\vdash \mathrm{P}^{j}, \mathrm{P}$ is equivalent to $j\left(c_{j}\right) \prod_{i \leqq n} A_{i} \supset z$. Now either the latter simple implication $\in \mathfrak{S}$ or it has a normal evaluation $v$ with respect to $J_{d-1}$. In the former case, $\vdash \mathrm{P}$. In the latter case, a normal evaluation of $\mathrm{P}$ with respect to $J_{d}$ can be constructed in terms of $v$.

Suppose, on the other hand, that no $\mathrm{P}^{i}$ fulfils $J_{d-1}$. Now for each $i$ $(1 \leqq i \leqq d)$ there is a normal evaluation $v_{i}$ with respect to $J_{d-1}$ of the $i$ th $\mathrm{P}^{j}$. Then a normal evaluation of $\mathrm{P}$ with respect to $J_{d}$ can be constructed by mapping each p-variable $\mathrm{q}$ into $\alpha_{M}\left(\left(v_{1}(\mathrm{q}), \cdots, v_{d}(\mathrm{q})\right)\right)$ where $M$ is $J_{d-1}^{(d)}$.

5.3.2. Suppose that a member of $K$ is a $p$-variable but no such $p$-variable occurs in a member of form (ii)-(vi). Then the lemma is easily established under the respective subcases that every member of $\mathrm{K}$ is a $\mathrm{p}$-variable or a member of $\mathrm{K}$ is not a $\mathrm{p}$-variable.

5.3.3. Suppose that $\mathrm{p}$ is a member of $\mathrm{K}$ and a member $\mathrm{A}_{j}$ contains $\mathrm{p}$ and is not a p-variable.

(15) For the case of empty $\Delta$, the corresponding proposition for $\left(R_{1}\right) \Pi_{\Gamma}$ is stated, without proof, by Jaśkowski.

${ }^{(16)}$ This lemma and proof were supplied by the author after several unsuccessful attempts to establish the "Lemma" of $[10$, p. 60$]$ with the aid of Jaskowski's rough outline. 
If $\mathrm{A}_{j}$ is $7 \mathrm{p}$, then $\vdash \mathrm{P}$.

If $A_{j}$ is $a \supset p, a \supset b \vee p, a \supset p \vee b,(a \supset b) \supset p$ or a \& b $\supset p$, then the lemma follows in view of its validity for $j(\mathrm{p}) \prod_{i \leqq n} \mathrm{~A}_{i} \supset$ z. $\supset z$.

If $A_{j}$ is $\mathrm{p} \supset \mathrm{a}$, then the lemma follows in view of its validity for $j(\mathrm{a}) \prod_{i \leqq n} \mathrm{~A}_{i}$

If $A_{j}$ is $\mathrm{p} \supset \mathrm{a} \vee \mathrm{b}$, then the lemma follows in view of its validity for $j(\mathrm{a}) \prod_{i \leqq n} \mathrm{~A}_{i} \supset \mathrm{z}$ and for $j(\mathrm{~b}) \prod_{i \leqq n} \mathrm{~A}_{i} \supset \mathrm{z}$.

If $A_{j}$ is $(p \supset a) \supset b, p \& a \supset b$ or $a \& p \supset b$, then the lemma follows in view of its validity for $j(\mathrm{a} \supset \mathrm{b}) \prod_{i \leqq n} \mathrm{~A}_{i} \supset z$.

If $A_{j}$ is $(a \supset p) \supset b$, then the lemma follows in view of its validity for $j$ (b) $\prod_{i \leqq n} A_{i} \supset z$.

5.4. THEOREM. $\left\{J_{i}\right\}$ is a regular characteristic sequence for $\mathfrak{S}$.

Proof. Use Corollary 4.4, Theorem 5.2, and Lemma 5.3, the last in conjunction with Theorems 3.3 and 5.2.

In addition to being a characteristic sequence for $\mathfrak{S},\left\{J_{i}\right\}$ affords a decision procedure for the Heyting propositional calculus. Thus, for an arbitrary $\mathrm{p}$-formula $\mathrm{P}$, an interdeducible simple implication $\mathrm{P}^{*}$ can be found by an effective procedure. By an effective procedure, it can be determined whether or not $\mathrm{P}^{*}$ fulfils $J_{d}$, where $d$ is the degree of the premise of $\mathrm{P}^{*}$, and consequently whether or not $\mathrm{P}^{*}$, and therefore $\mathrm{P}$, is in $\mathfrak{S}$. (It should be noted that the number $\nu(i)$ of elements in $J_{i}$ is a rapidly increasing function of $i\left({ }^{17}\right)$. For this reason, the latter step in the decision procedure is, in general, too long to be practical.)

\section{PART II}

6. Proof that, classically, the Heyting propositional calculus is incomplete with respect to realizability. The following intuitive symbolism will be used henceforth.

\begin{tabular}{ll} 
Intuitiye symbolism & Predicate or natural number denoted \\
\hline$P \rightarrow Q$ & $P$ (materially) implies $Q$ \\
$P \& Q$ & $P$ and $Q$ \\
$P \vee Q$ & $P$ or $Q$ \\
$P \equiv Q$ & $(P \rightarrow Q) \&(Q \rightarrow P)$ \\
$(x) P$ & for all $x, P$ \\
$(E x) P$ & there exists $x$ such that $P$ \\
$\mu x P$ & \{the least $x$ such that $P$, if $(E x) P ;$ \\
$(x)_{R} P$ & $(x)(R \rightarrow P)$ \\
$(E x)_{R} P$ & $(E x)(R \& P)$
\end{tabular}

(17) We have $\nu(0)=2, \nu(i+1)=(\nu(i))^{i+1}+1$, so that $J_{5}$ already has more than $10^{60}$ elements. 
Intuitive symbolism Predicate or natural number denoted

$\mu x_{R} P$

$\lambda x_{1} \cdots x_{m} \cdot t$

$\mu x(R \& P)$

$(x)_{n}$

The function which $t$ is of $x_{1}, \cdots, x_{m}$ in that order $\left({ }^{18}\right)$

0 or the highest power of the $(n+1)$ th prime which divides $x$, according as $x$ is 0 or not $\left({ }^{19}\right)$

For any $m \geqq 1$ and any $z, x_{1}, \cdots, x_{m}$, we let $\{z\}\left(x_{1}, \cdots, x_{m}\right)$ be $U\left(\mu y T_{m}\left(z, x_{1}, \cdots, x_{m}, y\right)\right)$. This is undefined when not $(E y) T_{m}\left(z, x_{1}, \cdots\right.$, $\left.x_{m}, y\right)$. The notation appeared first in [11, footnote 7$]$. For the definitions and theory of the primitive recursive function $U$ and the primitive recursive predicate $T_{m}$, cf. $[12, \S \S 7,4]$ or $[17, \S \S 57,58,63,65]$.

We shall have available a primitive recursive function $S_{n}^{m}$ of $m+1$ variables (for each $m \geqq 1$ and $n \geqq 1$ ) with the following property. If $e$ defines recursively $\phi\left(y_{1}, \cdots, y_{m}, x_{1}, \cdots, x_{n}\right)$ as a function of $m+n$ variables, and $k_{1}, \cdots, k_{m}$ are fixed natural numbers, then $S_{n}^{m}\left(e, k_{1}, \cdots, k_{m}\right)$ defines recursively $\phi\left(k_{1}, \cdots, k_{m}, x_{1}, \cdots, x_{n}\right)$ as a function of the remaining variables $x_{1}, \cdots, x_{n}\left({ }^{20}\right)$.

For any natural number $e$ and n-formula $\mathrm{F}$, "e $r \mathrm{~F}$ " shall mean that $e$ realizes $\mathrm{F}$. For any n-formula $\mathrm{F}$, " $r \mathrm{~F}$ " shall mean that $\mathrm{F}$ is realizable, i.e. that $(E e) e r F$.

If " $x$," " $x_{1}$, " " $y$, " . . represent certain natural numbers intuitively, then " $x$," " $x_{1}, " ~ y, " \cdots$ shall represent the corresponding numerals, and conversely.

If $\mathrm{x}_{1}, \cdots, \mathrm{x}_{m}$ are distinct $\mathrm{n}$-variables ( $\mathrm{p}$-variables), and if $\mathrm{F}\left(\mathrm{x}_{1}, \cdots, \mathrm{x}_{m}\right)$ " is explicitly introduced to stand for a certain term or $n$-formula (a certain $\mathrm{p}$-formula), thereafter for any other appropriate set of terms (of $\mathrm{n}$-formulas or p-formulas) $t_{1}, \cdots, t_{m}, " F\left(t_{1}, \cdots, t_{m}\right)$ " shall stand for the result of substituting $t_{1}, \cdots, t_{m}$ for the free occurrences (for the occurrences) of $\mathrm{x}_{1}, \cdots, \mathrm{x}_{m}$, respectively, throughout $\mathrm{F}\left(\mathrm{x}_{1}, \cdots, \mathrm{x}_{m}\right)$.

As we remarked in the introduction, it follows from Nelson's Theorem 1 that the Heyting propositional calculus is consistent with respect to realizability. On the other hand, for the intuitionist, the question as to its completeness remains open. In Theorem 6.1, however, we exhibit a p-formula not in $\mathfrak{S}$, whose realizability follows from the law of double negation for a particular predicate of the form $(E c) Q(b, c)$ with $Q$ primitive recursive. Hence, classically, the Heyting propositional calculus is incomplete with respect to realizability.

(18) This notation is taken from [1]. Our use differs from that of Church, however, in that his $\lambda$ was defined only for functions of one variable, and functions of $m$ variables were obtained by iteration.

(19) The notation appeared first in $\left[12\right.$, p. 50]. Here $(x)_{n}$ is used in the sense of [15] or $[17, \$ 45, \# 19]$; it is primitive recursive.

(20) Cf. $[11$, p. 153$]$ or $[17, \S 65$, Theorem XXIII $]$. 


\subsection{Theorem. Classically, $\mathfrak{B} \subset \mathfrak{S}$.}

Proof. Let $\left.\mathrm{D}=\operatorname{def}\rceil \mathrm{a}_{0} \vee\right\urcorner \mathrm{a}_{1}$, where $\mathrm{a}_{0}$ and $\mathrm{a}_{1}$ are distinct $\mathrm{p}$-variables, and $\left.\mathrm{P}\left(\mathrm{a}_{0}, \mathrm{a}_{1}\right)=\operatorname{def}((\neg \neg \mathrm{D} \supset \mathrm{D}) \supset\rceil 7 \mathrm{D} \vee \neg \mathrm{D}\right) \supset \neg \neg \mathrm{D} \vee \neg \mathrm{D}$. Let $f_{0}$ define recursively $\lambda b \cdot 2^{0} \cdot 3^{0}, f_{1}$ define recursively $\lambda b \cdot 2^{1} \cdot 3^{0}$, and $f=2^{f_{0}} \cdot 3^{f_{1}}$. Define as follows the primitive recursive predicates $R$ and $S$ :

$$
\begin{aligned}
R(b, c) & \equiv \operatorname{def}(E j)_{j<2}\left(T_{1}\left(b,(f)_{j},(c)_{j}\right) \&\left(U\left((c)_{j}\right)\right)_{0}=0\right) \&(c)_{2}=2^{0} \cdot 3^{0}, \\
S(b, c) & \equiv \operatorname{def}(j)_{j<2}\left(T_{1}\left(b,(f)_{j},(c)_{j}\right) \&\left(U\left((c)_{j}\right)\right)_{0}=1\right) \&(c)_{2}=2^{1} \cdot 3^{0} .
\end{aligned}
$$

Let $e$ define recursively $\lambda b \cdot(\mu c(R(b, c) \bigvee S(b, c)))_{2}$. We shall show that if

$$
(b)(\text { not not }(E c)(R(b, c) \vee S(b, c)) \rightarrow(E c)(R(b, c) \vee S(b, c))) \text {, }
$$

then, for arbitrary $n$-formulas $F_{0}$ and $F_{1}$,

$$
\left(z_{0}\right) \cdots\left(z_{p}\right) \text { er } \mathrm{P}\left(\mathrm{F}_{0}^{*}, \mathrm{~F}_{1}^{*}\right),
$$

where $z_{0}, \cdots, z_{p}$ are the distinct free $n$-variables of $P\left(F_{0}, F_{1}\right)$ in order of first free occurrence, and $F_{0}^{*}$ and $F_{1}^{*}$ result from $F_{0}$ and $F_{1}$ respectively by substituting $z_{0}, \cdots, z_{p}$ for $z_{0}, \cdots, z_{p}$. Thus the realizability of $\mathrm{P}\left(\mathrm{a}_{0}, \mathrm{a}_{1}\right)$ follows from the classically true proposition (1).

We note the following facts pertaining to an arbitrary closed n-formula $\mathrm{E}$. It follows directly from $[13, \S 5]$ that if $\mathrm{E}$ is unrealizable, then $7 \mathrm{E}$ is realized by any natural number, in particular by 0 . Thence, using also [13, p. 114 (c)], we have $($ not $r \mathrm{E}) \equiv r\rceil \mathrm{E}$; and hence $($ not not $r \mathrm{E}) \equiv(\operatorname{not} r\rceil \mathrm{E}) \equiv r\rceil 7 \mathrm{E}$.

For convenience, let us denote $\left.\rceil \mathrm{F}_{0}^{*} \vee\right\urcorner \mathrm{F}_{1}^{*}$ by "G."

Now assume (1). We show that, if

$$
\operatorname{br}(\neg \neg \mathrm{G} \supset \mathrm{G}) \supset \neg \neg \mathrm{G} \vee \neg \mathrm{G} \text {, }
$$

then

$$
\{e\}(b) r \neg 7 \mathrm{G} \vee \neg \mathrm{G} \text {; }
$$

i.e. that (2) holds. To show this, assume (3) and

$$
\operatorname{not}(E c)(R(b, c) \vee S(b, c)) \text {. }
$$

Assume

$$
r \mathrm{G} \text {, }
$$

so that $r\rceil 7 \mathrm{G}$ but not $r\rceil \mathrm{G}$. From (6), $r\urcorner \mathrm{F}_{0}^{*} \vee r \neg \mathrm{F}_{1}^{*}$. Therefore $\left(2^{0} \cdot 3^{0} r \mathrm{G}\right)$ $\vee\left(2^{1} \cdot 3^{0} r \mathrm{G}\right)$. Therefore $\left(f_{0} r \neg \neg \mathrm{G} \supset \mathrm{G}\right) \vee\left(f_{1} r \neg \neg \mathrm{G} \supset \mathrm{G}\right)$. Therefore either $(E c)\left(T_{1}\left(b,(f)_{0}, c\right) \&(U(c))_{0}=0\right)$ or $(E c)\left(T_{1}\left(b,(f)_{1}, c\right) \&(U(c))_{0}=0\right)$. Hence $(E c) R(b, c)$. In view of $(5)$, we have

$$
\text { not } r \mathrm{G}
$$

with (6) discharged. Then not $r\urcorner 7 \mathrm{G}$ but $r\urcorner \mathrm{G}$, so that $f_{0}$ and $f_{1}$ each realize 
$\neg \neg$ G $\supset$. Therefore $(E c)\left(T_{1}\left(b,(f)_{0}, c\right) \&(U(c))_{0}=1\right) \&(E c)\left(T_{1}\left(b,(f)_{1}, c\right)\right.$ $\left.\&(U(c))_{0}=1\right)$. Hence $(E c) S(b, c)$. In view of $(5)$, we have not not $(E c)(R(b, c)$ $\bigvee S(b, c))$ with (5) discharged. From (1), then, $(E c)(R(b, c) \bigvee S(b, c))$. Let $c=\mu c(R(b, c) \bigvee S(b, c))$. Then $R(b, c) \bigvee S(b, c)$. Assume

$$
R(b, c)
$$

and

$$
\text { not } r \mathrm{G} \text {. }
$$

Then $f_{0}$ and $f_{1}$ each realize $\neg 7 \mathrm{G} \supset \mathrm{G}$, so that $\left(T_{1}\left(b,(f)_{0},(c)_{0}\right) \rightarrow\left(U\left((c)_{0}\right)\right)_{0}\right.$ $=1) \&\left(T_{1}\left(b,(f)_{1},(c)_{1}\right) \rightarrow\left(U\left((c)_{1}\right)\right)_{0}=1\right)$. Therefore not $R(b, c)$. Thus not not $r \mathrm{G}$ with (9) discharged. It follows that $r \neg \neg \mathrm{G}$, so that $(c)_{2} r \neg \neg \mathrm{G} \vee \neg \mathrm{G}$. Assume

$$
S(b, c)
$$

and

$$
r \mathrm{G} \text {. }
$$

Then $\left.\left.\left(f_{0} r\right\urcorner \neg G \supset G\right) \vee\left(f_{1} r\right\rceil 7 G \supset G\right)$, so that $\left(T_{1}\left(b,(f)_{0},(c)_{0}\right) \rightarrow\left(U\left((c)_{0}\right)\right)_{0}\right.$ $=0) \vee\left(T_{1}\left(b,(f)_{1},(c)_{1}\right) \rightarrow\left(U\left((c)_{1}\right)\right)_{0}=0\right)$. Therefore not $S(b, c)$. Thus not $r \mathrm{G}$ with (11) discharged. It follows that $r\urcorner \mathrm{G}$, so that $\left.\left.(c)_{2} r\right\urcorner\right\rceil \mathrm{G} \vee \neg \mathrm{G}$. But $\{e\}(b)=(c)_{2}$, so that (4) holds with (8) and (10) discharged.

Thus, classically, $\mathrm{P}\left(\mathrm{a}_{0}, \mathrm{a}_{1}\right) \in \mathfrak{B}$. The proof is completed by showing that $\mathrm{P}\left(\mathrm{a}_{0}, \mathrm{a}_{1}\right)$ does not fulfil $J_{3}$, so that, in view of Theorem 5.4, $\mathrm{P}\left(\mathrm{a}_{0}, \mathrm{a}_{1}\right) \notin \mathfrak{S}$. Let $v\left(\mathrm{a}_{0}\right)$ and $v\left(\mathrm{a}_{1}\right)$ be $(((1),(0)),((0),(0)),((0),(0)))$ and $(((0),(1))$, $((0),(0)),((0),(0)))$ respectively. Then it can be shown that $v\left(\mathrm{P}\left(\mathrm{a}_{0}, \mathrm{a}_{1}\right)\right)=4$.

7. The completeness with respect to realizability of the $\supset$-less part of the Heyting propositional calculus.

7.1. Theorem. Every つ-less p-formula not in $\mathfrak{S}$ is equivalent to a disjunction $\sum_{j \leqq n} \mathrm{P}_{j}$ of $p$-formulas not in $\mathfrak{A}$.

Proof. A p-variable already has the required form. Assume that the theorem holds for $\supset$-less $\mathrm{p}$-formulas $\mathrm{P}$ and $\mathrm{Q}$; then it can be shown that the theorem holds for $\mathrm{P} \& \mathrm{Q}, \mathrm{P} \vee \mathrm{Q}$, and $\rceil \mathrm{P}$. In treating $\rceil \mathrm{P}$, note that $\rceil \mathrm{P} \in \mathfrak{X}$ $\rightarrow\rceil \mathrm{P} \in \mathfrak{S}$ (cf. Glivenko [3]).

7.2. LемMA. Let $\epsilon_{0}, \epsilon_{1}, \cdots$ be the sequence of (primitive recursive) functions of one variable such that $\epsilon_{0}$ is identically 0 and, for all $m>0, \epsilon_{m}(e)=\epsilon_{m-1}\left((e)_{1}\right)$ or $m$ according as $(e)_{0}$ is 0 or not. If $\mathrm{F}$ is a closed $n$-formula of the form $\sum_{i \leqq m} \mathrm{~F}_{i}$, then for all $e$,

$$
\operatorname{er} \mathrm{F} \rightarrow(E i)_{i \leqq m}\left(\epsilon_{m}(e)=i \& r \mathrm{~F}_{i}\right) .
$$

Proof. Use induction on $m$. 
7.3. For Theorem 7.4, let $x$ and $y$ be distinct n-variables, and for each $j$ let " $\mathrm{U}_{j}(\mathrm{x})$ " denote $\exists \mathrm{yV}_{j}(\mathrm{x}, \mathrm{y})$, where $\mathrm{V}_{j}$ is a predicate symbol expressing the primitive recursive predicate $T_{1}\left((x)_{j}, x, y\right)$. We are making the assumption that the predicate symbols $V_{j}$ are available in the number-theoretic formal system for convenience. However, this entails no loss of generality in the results; cf. [17, $\$ 82$ Lemma 47 and $\$ 49$ Corollary Theorem 27] (also [13, p. 116 first paragraph and p. 119 second paragraph from below $]$ ). The same remark applies to the use of the predicate symbol $\mathrm{A}$ in $\$ 8$.

7.4. Theorem. Let $\mathrm{P}\left(\mathrm{a}_{0}, \cdots, \mathrm{a}_{n}\right)$ be an arbitrary disjunction $\sum_{i \leqq m} \mathrm{P}_{i}$ of $p$-formulas not in $\mathfrak{A}$, where $\mathrm{a}_{0}, \cdots, \mathrm{a}_{n}$ are the distinct p-variables of $\mathrm{P}\left(\mathrm{a}_{0}, \cdots, \mathrm{a}_{n}\right)$. Then not $r \mathrm{P}\left(\mathrm{U}_{0}(\mathrm{x}), \cdots, \mathrm{U}_{n}(\mathrm{x})\right)$.

Proof. There exist (cf. 4.2) evaluating functions $v_{0}, \cdots, v_{m}$ with respect to $L_{1}$ such that $(i)_{i \leqq m} v_{i}\left(\mathrm{P}_{i}\right)=1$. Assume that $r \mathrm{P}\left(\mathrm{U}_{0}(\mathrm{x}), \cdots, \mathrm{U}_{n}(\mathrm{x})\right)$. Then there exists a general recursive function $\phi$ such that

$$
(x) \phi(x) r \mathrm{P}\left(\mathrm{U}_{0}(\mathrm{x}), \cdots, \mathrm{U}_{n}(\mathrm{x})\right) \text {. }
$$

For each $(i, j)(i \leqq m, j \leqq n)$, let the general recursive predicate $R_{i j}$ be given by

$$
R_{i j}(x) \equiv \operatorname{def} \epsilon_{m}(\phi(x))=i \& v_{i}\left(\mathrm{a}_{j}\right)=0 .
$$

Then (cf. [12, Theorem I]) there are natural numbers $f_{0}, \cdots, f_{n}$ such that, for each $j \leqq n$,

$$
\sum_{i \leqq m} R_{i j}(x) \equiv(E y) T_{1}\left(f_{j}, x, y\right)
$$

Let $f=p_{0}^{f_{0}} \cdots p_{n}^{f_{n}}$. In view of Lemma 7.2 and (1), there is a natural number $i^{*} \leqq m$ such that

$$
\epsilon_{m}(\phi(f))=i^{*} \& r \mathrm{Q}_{i^{*}},
$$

where $Q_{i}$ results from $P_{i}$ under the substitution of $\mathrm{U}_{0}(f), \cdots, \mathrm{U}_{n}(f)$ for $\mathrm{a}_{0}, \cdots, \mathrm{a}_{n}$. Then, using (2), for arbitrary $j \leqq n, v_{i}\left(\mathrm{a}_{j}\right)=0 \rightarrow \sum_{i \leqq m} R_{i j}(f)$; moreover $(i)\left(i \leqq m \& R_{i j}(f) \rightarrow i=i^{*} \& v_{i}\left(\mathrm{a}_{j}\right)=0\right)$, so that $\sum_{i \leqq m} R_{i j}(f) \rightarrow v_{i}{ }^{*}\left(\mathrm{a}_{j}\right)$ $=0$. Thus, using (3), $(j)_{j \leqq n}\left((E y) T_{1}\left((f)_{j}, f, y\right) \equiv v_{i}\left(a_{j}\right)=0\right)$. It follows (cf. $[13$, p. $114,(\mathrm{a}),(\mathrm{m})])$ that $(j)_{j \leqq n}\left(r \mathrm{U}_{j}(f) \equiv v_{i}{ }^{*}\left(\mathrm{a}_{j}\right)=0\right)$. Therefore, in obtaining $Q_{i}$ from $\mathrm{P}_{i^{*}}$, the closed $\mathrm{n}$-formula substituted for any $\mathrm{p}$-variable a is realizable or not according as $v_{i}(\mathrm{a})$ is 0 or 1 . Consequently (cf. [17, $\$ 82$ Example 3(a)]), inasmuch as $v_{i^{*}}\left(\mathrm{P}_{i^{*}}\right)=1$, not $r \mathrm{Q}_{i^{*}}$, contrary to (4). We conclude that not $r \mathrm{P}\left(\mathrm{U}_{0}(\mathrm{x}), \cdots, \mathrm{U}_{n}(\mathrm{x})\right)$.

Consider an arbitrary $\supset$-less p-formula $\mathrm{P}$ not in $\mathfrak{S}$. By Theorem 7.1, $\mathrm{P}$ is equivalent to a $\mathrm{p}$-formula $\mathrm{Q}$ which is a disjunction $\sum_{i \leqq m} \cdot \mathrm{P}_{i}$ of $\mathrm{p}$-formulas not in $\mathfrak{A}$. Substitute $\mathrm{n}$-formulas for the $\mathrm{p}$-variables of $\mathrm{P}$ and $\mathrm{Q}$ in such a way that $\mathrm{U}_{0}(\mathrm{x}), \cdots, \mathrm{U}_{n}(\mathrm{x})$ are substituted for the distinct $\mathrm{p}$-variables $\mathrm{a}_{0}, \cdots, \mathrm{a}_{n}$ 
of $Q$; let $\mathrm{P}^{*}$ and $\mathrm{Q}^{*}$ be the $\mathrm{n}$-formulas resulting under this substitution from $P$ and $Q$ respectively. In view of the consistency of the Heyting propositional calculus with respect to realizability, $r \mathrm{P}^{*} \supset \mathrm{Q}^{*}$. By Theorem 7.4, not $r \mathrm{Q}^{*}$; hence not $r \mathrm{P}^{*}$. We conclude that, for every $\supset$-less $\mathrm{p}$-formula $\mathrm{P}, \mathrm{P} \in \mathfrak{S}$ $\rightarrow \mathrm{P} \in \mathfrak{B}$. Because there is a decision procedure for the Heyting propositional calculus (cf. §5, end), we can conclude (intuitionistically) that $\mathrm{P} \in \mathfrak{P} \rightarrow \mathrm{P} \in \mathfrak{S}$. Combining this result with the consistency property, we have Theorem 7.5.

\subsection{Theorem. For any $\supset$-less p-formula $\mathrm{P}, \mathrm{P} \in \mathfrak{W} \equiv \mathrm{P} \in \mathfrak{B}$.}

\subsection{THEOREM. $\mathfrak{B}$ has no regular characteristic matrix.}

Proof. Let $M$ be an $n$-valued, regular characteristic matrix of $\mathfrak{B}$. We exhibit a $\mathrm{p}$-formula $\mathrm{P}$ of the form $\sum_{i \leqq m} \mathrm{P}_{i}$ whose members are not in $\mathfrak{A}$ and which fulfils $M$. (Gödel [6] gives a stronger p-formula of this form which serves the same purpose.) Then $P \in \mathfrak{B}$, contrary to Theorem 7.4.

Let $\mathrm{a}_{0}, \cdots, \mathrm{a}_{n}$ be distinct $\mathrm{p}$-variables. Let $\left(p_{0}, q_{0}\right), \cdots,\left(p_{m}, q_{m}\right)$ be the distinct ordered pairs of natural numbers $(p, q)$ such that $p<q \leqq n$. For all $i \leqq m$, let $\mathrm{P}_{\boldsymbol{i}}=\operatorname{def} \neg\left(\mathrm{a}_{p_{\boldsymbol{i}}} \& \mathrm{a}_{q_{i}}\right)$. Then $\sum_{i \leqq m} \mathrm{P}_{\boldsymbol{i}}$ is a p-formula $\mathrm{P}$ whose members are not in $\mathfrak{A}$. Consider an arbitrary evaluating function $v$ with respect to $M$. Since there is one more $\mathrm{p}$-variable in $\mathrm{P}$ than there are elements in $M, v$ must map at least one pair of distinct p-variables $\mathrm{a}_{p_{i}}, \mathrm{a}_{q_{i}}(i \leqq m)$ into the same element. For such a $p_{i}$ and $q_{i}, v\left(\mathrm{P}_{i}\right)=v\left(\neg\left(\mathrm{a}_{p_{i}} \& \neg \mathrm{a}_{p_{i}}\right)\right)$. In view of the consistency of the Heyting propositional calculus with respect to realizability, $\neg\left(\mathrm{a}_{p_{i}} \& \neg \mathrm{a}_{p_{i}}\right)$ and $\mathrm{P}_{i} \supset \mathrm{P}$ are in $\mathfrak{B}$, so that $v\left(\mathrm{P}_{i}\right)$ and $v\left(\mathrm{P}_{i} \supset \mathrm{P}\right)$ are designated, hence so is $v(\mathrm{P})$.

\section{Sets which contain $\mathfrak{P}$.}

8.1. Lemma. Hypothesis: $\mathrm{a}_{0}, \cdots, \mathrm{a}_{m}$ are p-variables; $v$ is an arbitrary evaluating function with respect to a matrix $L_{n}(n>0) ; x_{0}, \cdots, x_{p}$ are the distinct natural numbers, in ascending order, among $0,1, v\left(\mathrm{a}_{0}\right), \cdots, v\left(\mathrm{a}_{m}\right)$; $\epsilon$ is the mapping, defined over $\left\{x_{0}, \cdots, x_{p}\right\}$, which carries $x_{i}$ into $i ; v^{*}$ is any evaluating function with respect to $L_{m+2}$ such that $v^{*}\left(\mathrm{a}_{0}\right)=\epsilon\left(v\left(\mathrm{a}_{0}\right)\right), \cdots, v^{*}\left(\mathrm{a}_{m}\right)$ $=\epsilon\left(v\left(\mathrm{a}_{m}\right)\right) ; \mathrm{P}$ is any p-formula whose p-variables are among $\mathrm{a}_{0}, \cdots, \mathrm{a}_{m}$. Conclusion: $v^{*}(\mathrm{P})=\epsilon(v(\mathrm{P}))$.

Proof. We use induction corresponding to the inductive definition of $\mathrm{P}$. Basis: The lemma obviously holds if $P$ is one of the p-variables $a_{0}, \cdots, a_{m}$. Induction step: $1^{\circ}$ Let $\mathrm{P}$ be $\mathrm{A} \circ \mathrm{B}$, where $\circ$ is $\supset$, \&, or $V$. Now $v(\mathrm{P})=v(\mathrm{~A}) \circ_{L_{n}} v(\mathrm{~B})$, where by the induction hypothesis $v^{*}(\mathrm{~A})=\epsilon(v(\mathrm{~A}))$ and $v^{*}(\mathrm{~B})=\epsilon(v(\mathrm{~B}))$. Then $v(\mathrm{~A})=0 \bigvee v(\mathrm{~A})>v(\mathrm{~B})>0 \equiv v^{*}(\mathrm{~A})=0 \bigvee v^{*}(\mathrm{~A})>v^{*}(\mathrm{~B})$ $>0$. By referring to 4.2 , we can verify that $v^{*}(\mathrm{~A}) O_{L_{m+2}} v^{*}(\mathrm{~B})=\epsilon(v(\mathrm{~A})$ $\left.\mathrm{O}_{L_{n}} v(\mathrm{~B})\right)$, so that $v^{*}(\mathrm{P})=\epsilon(v(\mathrm{P}))$. $2^{\circ}$ Let $\mathrm{P}$ be $7 \mathrm{~A}$. Now $v(\mathrm{P})=7_{L_{n}} v(\mathrm{~A})$, where by the induction hypothesis $v^{*}(\mathrm{~A})=\epsilon(v(\mathrm{~A}))$. Then $v(\mathrm{~A})=1 \equiv v^{*}(\mathrm{~A})=1$. It follows from 4.2 that $\left.\rceil_{L_{m+2} v^{*}}(\mathrm{~A})=\epsilon(\rceil_{L_{n}} v(\mathrm{~A})\right)$, so that $v^{*}(\mathrm{P})=\epsilon(v(\mathrm{P}))$.

Let $\mathbb{R}$ be the set of $\mathrm{p}$-formulas for which $\left\{L_{n}\right\}$ is a characteristic sequence. 
8.2. Theorem. Let $\mathrm{P}$ be an arbitrary $p$-formula, and $\mathrm{a}_{0}, \cdots, \mathrm{a}_{m}$ be its distinct p-variables. Then $\mathrm{P} \in \mathbb{R} \equiv \mathrm{P}$ fulfils $L_{m+2}$.

Proof. Clearly, $\mathrm{P} \in \mathbb{R} \rightarrow \mathrm{P}$ fulfils $L_{m+2}$. Now, for any $n$, let $v$ be an evaluating function with respect to $L_{n}$ which maps $\mathrm{P}$ into $A_{L_{n}}$. Construct $\epsilon$ and $v^{*}$ as in the hypothesis of Lemma 8.1. Then $v^{*}(\mathrm{P})=\epsilon(v(\mathrm{P})) \in A_{L_{m+2}}$. Thus not P fulfils $L_{n} \rightarrow$ not P fulfils $L_{m+2}$. But the predicate P fulfils $L_{n}$ is effectively decidable; hence, intuitionistically, P fulfils $L_{m+2} \rightarrow \mathrm{P}$ fulfils $L_{n}$. We conclude that $\mathrm{P}$ fulfils $L_{m+2} \rightarrow \mathrm{P} \in \mathbb{R}$.

CoRollary. The predicate $\mathrm{P} \in \mathbb{R}$ is effectively decidable.

8.3. Definition. Let “ $\mathrm{B}(\mathrm{x})$ " denote $\exists \mathrm{yA}(\mathrm{x}, \mathrm{y}) \bigvee \bigvee \exists y \mathrm{~A}(\mathrm{x}, \mathrm{y})$, where $\mathrm{A}$ is a predicate symbol expressing the primitive recursive predicate $T_{1}(x, x, y)$, and $x$ and $y$ are distinct $n$-variables (cf. 7.3). The $n$-formula $B(x)$ is unrealizable (cf. $\left[17, \S 82\right.$ Theorem 63 (i) ]). We now define the n-formulas $F_{n}(x)$ and $\mathrm{G}_{n}(\mathrm{x})(n=1,2, \cdots)$, in each of which $\mathrm{x}$ is the sole free $\mathrm{n}$-variable:

$$
\mathrm{F}_{1}(\mathrm{x})=\operatorname{def} \neg \mathrm{B}(\mathrm{x}), \quad \mathrm{G}_{1}(\mathrm{x})=\operatorname{def} \forall \mathrm{z}(\mathrm{z}<\mathrm{x} \supset \mathrm{B}(\mathrm{z})),
$$

where $\mathrm{z}$ does not occur in $\mathrm{B}(\mathrm{x})$; for all $n>0$,

$$
\begin{aligned}
& \mathrm{G}_{n+1}(\mathrm{x})=\operatorname{def} \forall \mathrm{z}\left(\mathrm{z}<\mathrm{x} \supset \mathrm{F}_{n}(\mathrm{z}) \vee \mathrm{G}_{n}(\mathrm{z})\right), \\
& \mathrm{F}_{n+1}(\mathrm{x})=\operatorname{def} \mathrm{G}_{n+1}(\mathrm{x}) \supset \mathrm{F}_{n}(\mathrm{x}) \vee \mathrm{G}_{n}(\mathrm{x}),
\end{aligned}
$$

where $z$ does not occur in $F_{n}(x) \vee G_{n}(x)$.

We shall make frequent use of the fact that any $n$-formula, deducible by means of the intuitionistic predicate calculus with equality and the Peano axioms from realizable $\mathrm{n}$-formulas, is realizable (cf. [23, Theorem 1] or $[17, \S 82$ Theorem 62 (a) ]). For the present use of " $\vdash$," cf. 3.1.

8.4. Lemma. For all $n>0$,

$$
\begin{aligned}
& \vdash \neg \mathrm{F}_{n+1}(\mathrm{x}), \\
& \vdash \neg \mathrm{G}_{n}(\mathrm{x}), \\
& \text { classically, not } r \mathrm{~F}_{n}(\mathrm{x}) \vee \mathrm{G}_{n}(\mathrm{x}) .
\end{aligned}
$$

Proof. First, we establish $\vdash\urcorner 7 \mathrm{G}_{1}(\mathrm{x})$ by formal induction. Thus $\vdash \neg 7 \mathrm{G}_{1}(0)$. Moreover, noting $\left.\vdash \forall \mathrm{x}\right\rceil 7 \mathrm{~B}(\mathrm{x})$, we have $\rceil 7 \mathrm{G}_{1}(\mathrm{x}) \vdash \neg \neg \forall \mathrm{z}(\mathrm{z}$ $<\mathrm{x} \supset \mathrm{B}(\mathrm{z})) \& \neg \neg \mathrm{B}(\mathrm{x}) \vdash \neg\rceil(\forall \mathrm{z}(\mathrm{z}<\mathrm{x} \supset \mathrm{B}(\mathrm{z})) \& \mathrm{~B}(\mathrm{x})) \vdash \neg\rceil \mathrm{G}_{1}\left(\mathrm{x}^{\prime}\right)$, with $\mathrm{x}$ held constant. Next, note that for all $n<0$,

$$
\vdash \mathrm{G}_{n}(\mathrm{x}) \supset \forall \mathrm{w}\left(\mathrm{w} \leqq \mathrm{x} \supset \mathrm{G}_{n}(\mathrm{w})\right) ;
$$

hence $\vdash \mathrm{G}_{n}(\mathrm{x}) \supset \mathrm{G}_{n+1}(\mathrm{x})$; hence $\neg 7 \mathrm{G}_{n}(\mathrm{x}) \vdash \neg \neg \mathrm{G}_{n+1}(\mathrm{x})$. So (2) holds. Furthermore, for all $n>0,7 \neg \mathrm{G}_{n}(\mathrm{x}) \vdash \neg \neg \mathrm{F}_{n+1}(\mathrm{x})$; hence (1) holds.

To prove (3), we use induction on $n$.

BAsIs. Note that $\vdash ך F_{1}(x)$ and $\rceil F_{1}(x), F_{1}(x) \vee G_{1}(x) \vdash G_{1}(x)$, so that 
$\mathrm{F}_{1}(\mathrm{x}) \bigvee \mathrm{G}_{1}(\mathrm{x}) \vdash \mathrm{G}_{1}(\mathrm{x}) \vdash \mathrm{B}(\mathrm{x})$. Therefore not $r \mathrm{~F}_{1}(\mathrm{x}) \vee \mathrm{G}_{1}(\mathrm{x})$.

INDUCTION STEP. For $n>0$, assume that

$$
r \mathrm{~F}_{n+1}(\mathrm{x}) \vee \mathrm{G}_{n+1}(\mathrm{x}) \text {. }
$$

Then there exists a general recursive function $\phi$ such that $(x) \phi(x) r \mathrm{~F}_{n+1}(x)$ $\vee \mathrm{G}_{n+1}(\mathrm{x})$.

Now assume also

$$
(E x)(y)_{y \geqq x}(\phi(y))_{0}=0 .
$$

For such an $x,(y)_{y \geq x}(\phi(y))_{1} r \mathrm{~F}_{n+1}(y)$. Let $e$ define recursively $\lambda y a \cdot(\phi(y))_{1}$. Consider any $y$. For all $a$, if $a r y \geqq x$, then $y \geqq x\left({ }^{21}\right)$, hence $\left\{S_{1}^{1}(e, y)\right\}(a) r \mathrm{~F}_{n+1}(y)$. Thus

$$
r \forall \mathrm{y}\left(\mathrm{y} \geqq \mathrm{x} \supset \mathrm{F}_{n+1}(\mathrm{y})\right)
$$

Note that

$$
\vdash \mathrm{F}_{n+1}(\mathrm{x}) \& \mathrm{G}_{n+1}(\mathrm{x}) \supset \mathrm{G}_{n+1}\left(\mathrm{x}^{\prime}\right) .
$$

Now (holding $x$ constant) $x<x, G_{n+1}(x) \vdash[(4)] G_{n+1}\left(x^{\prime}\right)$, and $x \geqq x, G_{n+1}(x)$, $\forall \mathrm{y}\left(\mathrm{y} \geqq \mathrm{x} \supset \mathrm{F}_{n+1}(\mathrm{y})\right) \vdash \mathrm{F}_{n+1}(\mathrm{x}) \& \mathrm{G}_{n+1}(\mathrm{x}) \vdash[(8)] \mathrm{G}_{n+1}\left(\mathrm{x}^{\prime}\right)$. Hence

$$
\mathrm{G}_{n+1}(\mathrm{x}), \forall \mathrm{y}\left(\mathrm{y} \geqq \mathrm{x} \supset \mathrm{F}_{n+1}(\mathrm{y})\right), \mathrm{G}_{n+1}(\mathrm{x}) \vdash \mathrm{G}_{n+1}\left(\mathrm{x}^{\prime}\right)
$$

with $x$ held constant. From (9) and $\vdash \mathrm{G}_{n+1}(0)$ by formal induction, $\mathrm{G}_{n+1}(\mathrm{x})$, $\forall \mathrm{y}\left(\mathrm{y} \geqq \mathrm{x} \supset \mathrm{F}_{n+1}(\mathrm{y})\right) \vdash \forall \mathrm{xG}_{n+1}(\mathrm{x})$. But $\forall \mathrm{xG}_{n+1}(\mathrm{x}) \vdash \forall \mathrm{x}\left(\mathrm{F}_{n}(\mathrm{x}) \bigvee \mathrm{G}_{n}(\mathrm{x})\right)$. Hence $\forall \mathrm{y}\left(\mathrm{y} \geqq \mathrm{x} \supset \mathrm{F}_{n+1}(\mathrm{y})\right) \vdash \mathrm{G}_{n+1}(\mathrm{x}) \supset \forall \mathrm{x}\left(\mathrm{F}_{n}(\mathrm{x}) \vee \mathrm{G}_{n}(\mathrm{x})\right) \vdash \neg \neg \mathrm{G}_{n+1}(\mathrm{x}) \supset \neg \neg \forall \mathrm{x}$ $\left(\mathrm{F}_{n}(\mathrm{x}) \vee \mathrm{G}_{n}(\mathrm{x})\right)$. Then, using (7) and (2), $r \neg \neg \forall \mathrm{x}\left(\mathrm{F}_{n}(\mathrm{x}) \vee \mathrm{G}_{n}(\mathrm{x})\right)$, contradicting the induction hypothesis (noting [13, p. 114 (f)]). By reductio a d absurdum, not $(E x)(y)_{\nu \geqq x}(\phi(y))_{0}=0$, with (6) discharged.

Thence classically

$$
(x)(E y)_{y \geqq x}(\phi(y))_{0} \neq 0 .
$$

But then $\lambda x \cdot \mu y_{y \geq x}(\phi(y))_{0} \neq 0$ is a general recursive function $\psi$. For arbitrary $x, \psi(x) \geqq x$; hence, letting $y=\psi(x)$, we have $0 r y \geqq x$ and $(\psi(x))_{1} r \mathrm{G}_{n+1}(y)$; thus $\left.(2 \exp \psi(x)) \cdot\left(3 \exp 2^{0} \cdot 3^{(\psi(x))}\right) r\right) \exists \mathrm{y}\left(\mathrm{y} \geqq \mathrm{x} \& \mathrm{G}_{n+1}(\mathrm{y})\right)$. Therefore $r \exists \mathrm{y}(\mathrm{y} \geqq \mathrm{x}$ \& $\left.\mathrm{G}_{n+1}(\mathrm{y})\right)$. But $\exists \mathrm{y}\left(\mathrm{y} \geqq \mathrm{x} \& \mathrm{G}_{n+1}(\mathrm{y})\right) \vdash[\mathrm{cf} .(4)] \mathrm{G}_{n+1}(\mathrm{x}) \vdash \mathrm{F}_{n}(\mathrm{x}) \backslash \mathrm{G}_{n}(\mathrm{x})$, so that $r \mathrm{~F}_{n}(\mathrm{x}) \vee \mathrm{G}_{n}(\mathrm{x})$, contradicting the induction hypothesis. By reductio ad absurdum not $r \mathrm{~F}_{n+1}(\mathrm{x}) \vee \mathrm{G}_{n+1}(\mathrm{x})$, with (5) discharged.

This completes the induction step.

Henceforth, we shall denote $F_{i}(x)$ and $G_{i}(x)$ by " $F_{i}$ " and " $G_{i}$ " for all $i>0$. Also, let $\mathrm{F}_{0}=\operatorname{def} 0=0$.

8.5. Lemma. For all $i$ and $j(i>j>0)$,

(21) Here we are assuming that $y \geqq x$ is a prime $n$-formula, but the proof could be similarly constructed under alternative formalizations (cf. 7.3). 


$$
\begin{aligned}
& \vdash \mathrm{F}_{j} \supset \mathrm{F}_{i}, \\
& \vdash \mathrm{F}_{i} \supset \mathrm{F}_{j} \sim \mathrm{F}_{j}, \\
& \vdash \mathrm{F}_{i} \& \mathrm{~F}_{j} \sim \mathrm{F}_{j}, \\
& \vdash \mathrm{F}_{i} \vee \mathrm{F}_{j} \sim \mathrm{F}_{i} .
\end{aligned}
$$

Proof. (11) is immediate. To prove (12), note

$$
\begin{aligned}
& i>j>0 \rightarrow \vdash \mathrm{G}_{j} \supset \mathrm{F}_{i}, \\
& j>0 \rightarrow \vdash \mathrm{G}_{j} \supset \mathrm{F}_{j} \sim \mathrm{F}_{j}
\end{aligned}
$$

(using (2) and $\vdash \neg \mathrm{F}_{1}$ and noting that $\vdash \neg \neg \neg \mathrm{B}(\mathrm{x}) \supset \neg \mathrm{B}(\mathrm{x})$, in establishing (16) for $j=1)$. Hence if $i>j>0, \vdash[(15)]\left(\mathrm{F}_{i} \supset \mathrm{F}_{j}\right) \supset\left(\mathrm{G}_{j} \supset \mathrm{F}_{j}\right) \vdash[(16)]$ $\left(F_{i} \supset F_{j}\right) \supset F_{j}$. We obtain (13) and (14) directly from (11).

8.6. Definition. For each $n>0$, define as follows the set $V_{n}$ of $n$-formulas: $1^{\circ} \mathrm{F}_{0}, \cdots, \mathrm{F}_{n} \in V_{n} ; 2^{\circ}$ if $\mathrm{A}$ and $\mathrm{B} \in V_{n}$, then $\mathrm{A} \circ \mathrm{B}$ and $\rceil \mathrm{A} \in V_{n}$, where $\circ$ is $\supset, \&$, or $\bigvee ; 3^{\circ}$ all members of $V_{n}$ are given by $1^{\circ}$ and $2^{\circ}$. Let the mapping $\epsilon_{n}$ be defined as follows over $V_{n}: 1^{\circ} \epsilon_{n}\left(\mathrm{~F}_{i}\right)=i(i=0, \cdots, n)$; $2^{\circ} \epsilon_{n}(\mathrm{~A} \circ \mathrm{B})=\epsilon_{n}(\mathrm{~A}) \circ_{L_{n}} \epsilon_{n}(\mathrm{~B})$, where $\circ \supset$ is,$\&$, or $\bigvee$, and $\left.\epsilon_{n}(\neg \mathrm{A})=\right\rceil_{L_{n}} \epsilon_{n}(\mathrm{~A})$.

\subsection{Lemma. For all $n>0, \epsilon_{n}(\mathrm{~F})=k \rightarrow \vdash \mathrm{F} \sim \mathrm{F}_{k}$ for every $\mathrm{F}$ in $V_{n}$.}

Proof. (We shall write “ $\epsilon$ " for $\epsilon_{n}$.) The lemma obviously holds for $F_{0}, \cdots, F_{n}$. If by the induction hypothesis the lemma holds for $A$ and $B$, then it holds for $\mathrm{A} \supset \mathrm{B}, \mathrm{A} \& \mathrm{~B}, \mathrm{~A} \vee \mathrm{B}$ and $7 \mathrm{~A}$. Thus, let $\epsilon(\mathrm{A})=i, \epsilon(\mathrm{B})=j$. Note that $\vdash F_{0}$ and refer to 4.2 for the operations of $L_{n}$.

If $i=0$, then $\epsilon(\mathrm{A} \supset \mathrm{B})=j, \epsilon(\mathrm{A} \& \mathrm{~B})=j, \epsilon(\mathrm{A} \vee \mathrm{B})=i$ and $\vdash \mathrm{A} \supset \mathrm{B} \sim \mathrm{F}_{0} \supset \mathrm{F}_{j}$ $\sim \mathrm{F}_{j}, \vdash \mathrm{A} \& \mathrm{~B} \sim \mathrm{F}_{0} \& \mathrm{~F}_{j} \sim \mathrm{F}_{j}, \vdash \mathrm{A} \vee \mathrm{B} \sim \mathrm{F}_{0} \vee \mathrm{F}_{j} \sim \mathrm{F}_{0}$. Now let $i>0$. If $j=0$, then $\epsilon(\mathrm{A} \supset \mathrm{B})=0, \epsilon(\mathrm{A} \& \mathrm{~B})=i, \epsilon(\mathrm{A} \vee \mathrm{B})=0$ and $\vdash \mathrm{A} \supset \mathrm{B} \sim \mathrm{A} \supset \mathrm{F}_{0} \sim \mathrm{F}_{0}$, $\vdash \mathrm{A} \& \mathrm{~B} \sim \mathrm{F}_{i} \& \mathrm{~F}_{0} \sim \mathrm{F}_{i}, \vdash \mathrm{A} \vee \mathrm{B} \sim \mathrm{A} \vee \mathrm{F}_{0} \sim \mathrm{F}_{0} ;$ if $i>j>0$, then $\epsilon(\mathrm{A} \supset \mathrm{B})=j$, $\epsilon(\mathrm{A} \& \mathrm{~B})=j, \epsilon(\mathrm{A} \vee \mathrm{B})=i$ and $\vdash \mathrm{A} \supset \mathrm{B} \sim \mathrm{F}_{i} \supset \mathrm{F}_{j} \sim[8.5] \mathrm{F}_{j}, \vdash \mathrm{A} \& \mathrm{~B} \sim \mathrm{F}_{i} \& \mathrm{~F}_{j}$ $\sim[8.5] \mathrm{F}_{j}, \vdash \mathrm{A} \vee \mathrm{B} \sim \mathrm{F}_{i} \vee \mathrm{F}_{j} \sim[8.5] \mathrm{F}_{i}$; if $i \leqq j$, then $\epsilon(\mathrm{A} \supset \mathrm{B})=0, \boldsymbol{\epsilon}(\mathrm{A} \& \mathrm{~B})=i$, $\epsilon(\mathrm{A} \vee \mathrm{B})=j$ and $\vdash \mathrm{A} \supset \mathrm{B} \sim \mathrm{F}_{i} \supset \mathrm{F}_{j} \sim[8.5] \mathrm{F}_{0}, \vdash \mathrm{A} \& \mathrm{~B} \sim \mathrm{F}_{i} \& \mathrm{~F}_{j} \sim[8.5]$ $\mathrm{F}_{i}, \vdash \mathrm{A} \vee \mathrm{B} \sim \mathrm{F}_{i} \vee \mathrm{F}_{j} \sim[8.5] \mathrm{F}_{j}$

If $i=1$, then $\epsilon(\neg \mathrm{A})=0$ and $\vdash \neg \mathrm{A} \sim 7 \mathrm{~F}_{1} \sim[8.3] \mathrm{F}_{0}$. If $i=0$, then $\epsilon(\neg \mathrm{A})$ $=1$ and $\vdash \neg \mathrm{A} \sim \neg \mathrm{F}_{0} \sim[8.3] \mathrm{F}_{1}$. If $i>1$, then $\left.\epsilon(\rceil \mathrm{A}\right)=1$ and $\vdash \neg \mathrm{A} \sim \neg \mathrm{F}_{i}$ $\sim[8.4,8.3] \mathrm{F}_{1}$.

\subsection{Theorem. Classically, $\mathfrak{B} \subset \Omega$.}

Proof. Let $\mathrm{P}$ be an arbitrary p-formula not in $\mathfrak{\Omega} ; \mathrm{a}_{0}, \cdots, \mathrm{a}_{m}$ be its distinct p-variables; $v$ be an evaluating function with respect to $L_{n}$ such that $v(\mathrm{P})>0$. For each $j \leqq m$, let $\mathrm{E}_{j}=\operatorname{def} \mathrm{F}_{v\left(\mathrm{a}_{j}\right)}$. Let $\mathrm{P}^{*}$ result from $\mathrm{P}$ under the substitution of $\mathrm{E}_{0}, \cdots, \mathrm{E}_{m}$ for $\mathrm{a}_{0}, \cdots, \mathrm{a}_{m}$. Then $\mathrm{P}^{*} \in V_{n}$, and for the mapping $\epsilon_{n}$ of 8.6, $\epsilon_{n}\left(\mathrm{P}^{*}\right)=v(\mathrm{P})$. From Lemma 8.7, therefore, $\vdash \mathrm{P}^{*} \sim \mathrm{F}_{v(\mathrm{P})}$. Thus $r \mathrm{P}^{*} \rightarrow r \mathrm{~F}_{v(\mathrm{P})}$; hence (classically) in view of (3), Lemma 8.4, not $r \mathrm{P}^{*}$. 
We conclude that $\mathrm{P} \in \mathfrak{B}$. Thus $\mathrm{P} \in \mathbb{R} \rightarrow \mathrm{P} \notin \mathfrak{B}$, from which $\mathrm{P} \in \mathfrak{B} \rightarrow \mathrm{P} \in \mathbb{R}$ follows intuitionistically in view of Corollary 8.2.

\subsection{Theorem. $\mathfrak{L} \nsubseteq \mathfrak{P}$.}

Proof. Let $\mathrm{a}_{0}$ and $\mathrm{a}_{1}$ be distinct p-variables. Referring to 4.2, we find that $\left(a_{0} \supset a_{1}\right) \bigvee\left(a_{1} \supset a_{0}\right) \in \mathfrak{R}$. By Theorem 7.4, this p-formula is not in $\mathfrak{B}$.

In view of Theorems 8.8 and $8.9, \mathfrak{B}$ is (classically) a proper subset of $\mathfrak{R}$. Now $\mathfrak{R}$ is a proper subset of $\mathfrak{A}$ (proper because $\mathrm{a} \vee \neg \mathrm{a}$ is in $\mathfrak{A}$ but not in $\mathfrak{R}_{2}$ ). Hence, through $\$ 6$ and Theorems 8.8 and 8.9 , we have established (classically) the following chain of proper set-inclusions:

$$
\mathfrak{E} \subset \mathfrak{B} \subset \mathfrak{R} \subset \mathfrak{A} \text {. }
$$

(Note that, intuitionistically, $\mathfrak{B}$ is a proper subset of $\mathfrak{A}$. Thus, it follows from $[17, \S 82$ Example 3 (a)] that $\mathfrak{B C} \mathfrak{A}$; moreover, we have referred in 8.3 to Kleene's example of an unrealizable $n$-formula obtained from $\mathrm{a} \vee \neg \mathrm{a}$; hence $\mathfrak{A} ণ \mathfrak{P}$.

\section{BIBLIOGRAPHY}

1. Alonzo Church, $A$ set of postulates for the foundation of logic, Ann. of Math. (2) vol. 33 (1932) pp. 346-366.

2. Gerhard Gentzen, Untersuchungen ibber das logische Schliessen, Math. Zeit. vol. 39 (19341935) pp. 176-210, 405-431.

3. V. Glivenko, Sur quelques points de la logique de $M$. Brouwer, Bulletin de la Classe des Sciences de l'Académie Royale de Belgique (5) vol. 15 (1929) pp. 183-188.

4. Kurt Gödel, Die Vollständigkeit der Axiome des logischen Funktionenkalküls, Monatshefte für Mathematik und Physik vol. 37 (1930) pp. 349-360.

5. - Über formal unentscheidbare Sätze der Principia Mathematica und verwandter Systeme I, Ibid. vol. 38 (1931) pp. 173-198.

6. - Zum intuitionistischen Aussagenkalul, Ergebnisse eines Mathematischen Kolloquiums, no. 4 (for 1931-1932, published 1933), p. 40.

7. Leon Henkin, An algebraic characterization of quantifiers, Fund. Math. vol. 37 (1949) pp. 63-74.

8. Arend Heyting, Die formalen Regeln der intuitionistischen Logik, Preuss. Akad. Wiss. Sitzungsber. (1930) pp. 42-56.

9. - Die formalen Regeln der intuitionistischen Mathematik, Ibid. pp. 57-71, 158-169.

10. Stanisław Jaskowski, Recherches sur le systeme de la logique intuitioniste, Actes du Congrès International de Philosophie Scientifique VI, Philosophie des mathématiques, Actualités Scientifiques et Industrielles, no. 393, Paris, Hermann, 1936, pp. 58-61. 155.

11. S. C. Kleene, On notation for ordinal numbers, J. Symbolic Logic vol. 3 (1938) pp. 150-

12. - Recursive predicates and quantifiers, Trans. Amer. Math. Soc. vol. 53 (1943) pp. 41-73.

13. - On the interpretation of intuitionistic number theory, J. Symbolic Logic vol. 10 (1945) pp. 109-124.

14. - On the intuitionistic logic, Proceedings of the $\mathrm{X}$ th International Congress of Philosophy (Amsterdam, Aug. 11-18, 1948), Amsterdam, 1949, no. 2, pp. 741-743.

15. — A symmetric form of Gödel's theorem, Neder. Akad. Wetensch. vol. 53 (1950) pp. 800-802; also in Indagationes Mathematicae vol. 12 (1950) pp. 244-246. 
16. - Recursive functions and intuitionistic mathematics, Proceedings of the International Congress of Mathematicians (Cambridge, Mass., Aug. 30-Sept. 6, 1950).

17. - Introduction to metamathematics, New York, Van Nostrand, Amsterdam, North Holland, and Gronigen, Noordhoff, forthcoming in 1952.

18. Leopold Löwenheim, Über Möglichkeiten im Relativkalkill, Math. Ann. vol. 76 (1915) pp. 447-470.

19. Jan Lukasiewicz, $O$ logice trojwartosciowej (On three-valued logic), Ruch filozoficzny (Lw6w) vol. 5 (1920) pp. 169-171.

20. Jan Lukasiewicz and Alfred Tarski, Untersuchungen ibber den Aussagenkalkiil, Comptes rendus des séances de la Société des Sciences et des Lettres de Varsovie, Cl. III, vol. 23 (1930) pp. 30-50.

21. J. C. C. McKinsey and Alfred Tarski, Some theorems about the sentential calculi of Lewis and Heyting, J. Symbolic Logic vol. 13 (1948) pp. 1-15.

22. Andrzej Mostowski, Proofs of non-deducibility in intuitionistic functional calculus, Ibid. vol. 13 (1948) pp. 204-207.

23. David Nelson, Recursive functions and intuitionistic number theory, Trans. Amer. Math. Soc. vol. 61 (1947) pp. 307-368.

24. Emil L. Post, Introduction to a general theory of elementary propositions, Amer. J. Math. vol. 43 (1921) pp. 163-185.

25. Thoralf Skolem, Einige Bemerkungen zur axiomatischen Begrïndung der Mengenlehre, Wissenschaftliche Vorträge gehalten auf dem $F$ ünften Kongress der Skandinavischen Mathematiker in Helsingfors vom 4. bis 7. Juli 1922, Helsingfors, 1923, pp. 217-232.

UNIVERSITY OF WisCONSIN, MADISON, Wis. 